\title{
TRACK: THE NEW BEAM DYNAMICS CODE *
}

\author{
V.N. Aseev, P.N. Ostroumov, E.S. Lessner and B. Mustapha ${ }^{\dagger}$, ANL, Argonne, IL 60439, USA
}

\begin{abstract}
The new ray-tracing code TRACK originally developed to fulfill the special requirements of the RIA accelerator systems is a general beam dynamics code. It is currently being used for the design and simulation of future proton and heavy-ion linacs at several Labs. This paper presents a general description of the code TRACK emphasizing its main new features and recent updates.
\end{abstract}

\section{INTRODUCTION}

Detailed design and beam dynamics simulations in the RIA accelerators required the development of a new computer code to track multi-component heavy-ion beams directly from the ECR ion source to the production targets. The code had to include a wide range of electromagnetic elements such as high-voltage accelerating tubes, RF resonators, electrostatic and magnetostatic multipoles with full three-dimensional fields, etc. In addition, the interaction of heavy-ion beams with material media such as strippers has to be included. For careful localization of beam losses the code had to calculate particle trajectories in the close vicinity of the apertures of electromagnetic devices. As a medium-energy SC linac the RIA driver contains a large number of accelerating, focusing and bending elements. Hence beam dynamics becomes quite sensitive to complex sets of errors and misalignments of these elements. The new code should be used for the statistical analysis of beam parameters obtained by tracking large numbers of particles through hundreds of accelerators each with randomly seeded errors. We have started developing the code TRACK [1] due to the lack of any code capable of simulating multiple-charge-state heavy-ion beam dynamics through such a wide range of 3D elements.

There are a number of available codes such as TRANSPORT [2], TRACE3D [3], COSY [4], GIOS [5] based on matrix formalism for the design and study of beam-optics systems. Some of them include detailed beam space charge calculations. However, none of these codes provide a full description of beam dynamics in all the elements of the RIA driver linac. A specially developed ray-tracing code such as TRACK is necessary for detailed studies of the accelerator and beam transport systems and for finalizing the design. A ray-tracing or particle-tracking code has many advantages over a matrix-based code, among these we mention: (i) The external field can be represented more accurately within the physical aperture of the device. (ii) Particle coordinates are known at any point in the space. (iii)

\footnotetext{
* Work supported by the U.S. Department of Energy, Office of Nuclear Physics, under Contract No. W-31-109-ENG-38.

† brahim@anl.gov
}

Non-electromagnetic elements, such as beam degraders or strippers can be accurately simulated. (iv) Beam space charge fields, especially for multi-component ion beams can be calculated with high accuracy. (v) Beam losses can be calculated in the presence of complex sets of field errors and device misalignments.

\section{GENERAL DESCRIPTION}

In the code TRACK, the transport of a charged particle is described by the equation of motion:

$$
\frac{d \vec{p}}{d t}=Q(\vec{E}+\vec{v} \times \vec{B}),
$$

where $\vec{p}$ is the particle momentum and $\mathrm{Q}$ is its charge, $\vec{E}=\vec{E}_{\text {ext }}+\vec{E}_{\text {int }}$ and $\vec{B}=\vec{B}_{\text {ext }}+\vec{B}_{\text {int }}$ are the sums of external and internal electric and magnetic fields, $\vec{v}$ is the particle velocity. The structure of the TRACK code and its ideology is close to the RAYTRACE code [6] except that the TRACK code has many additional features and capabilities. Unlike RAYTRACE, TRACK integrates the equations of motion of all tracked particles for a short distance and calculates space charge fields. The fourth order RungeKutta method is used for the integration of particle trajectory through an ion-optics device. TRACK uses the independent variable $z$ for tracking of phase-space coordinates of the particles $\left\{\mathrm{x}, \mathrm{x}^{\prime}=\mathrm{dx} / \mathrm{dz}, \mathrm{y}, \mathrm{y}^{\prime}=\mathrm{dy} / \mathrm{dz}, \beta=v / c, \phi\right\}$, where $v=|\vec{v}|, \phi$ is the particle phase with respect to the $\mathrm{RF}$ field at the given section of the accelerator.

As in the code RAYTRACE, when sufficiently small step sizes are used, the accuracy is limited only by the uncertainties in our knowledge of the magnetic and electric fields. The calculation of the space charge fields is performed in the curvilinear coordinate system of the reference particle (RP). Appropriate transformation of space charge forces from the curvilinear coordinate system to the input coordinate system is required. The set of equations used for the step-by-step integration routine is:

$$
\begin{gathered}
\frac{d x}{d z}=x^{\prime}, \frac{d y}{d z}=y^{\prime}, \frac{d \phi}{d z}=\frac{2 \pi f_{0} h}{\beta c}, \\
\frac{d x^{\prime}}{d z}=\chi \frac{Q}{A} \frac{h}{\beta \gamma}\left[\frac{h}{\beta c}\left(E_{x}-x^{\prime} E_{z}\right)+x^{\prime} y^{\prime} B_{x}\right. \\
\left.-\left(1+x^{\prime 2}\right) B_{y}+y^{\prime} B_{z}\right], \\
\frac{d y^{\prime}}{d z}=\chi \frac{Q}{A} \frac{h}{\beta \gamma}\left[\frac{h}{\beta c}\left(E_{y}-y^{\prime} E_{z}\right)-x^{\prime} y^{\prime} B_{y}\right. \\
\left.+\left(1+y^{\prime 2}\right) B_{x}-x^{\prime} B_{z}\right], \\
\frac{d \beta}{d z}=\chi \frac{Q}{A} \frac{h}{\beta \gamma^{3} c}\left(x^{\prime} E_{x}+y^{\prime} E_{y}+E_{z}\right),
\end{gathered}
$$

where $\gamma=1 / \sqrt{1-\beta^{2}}, h=1 / \sqrt{1+x^{\prime 2}+y^{\prime 2}}, \chi=$ $1 / m_{a} c^{2}, \mathrm{~A}$ is the mass number, $m_{a}$ is the atomic unit mass, 
$\mathrm{B}_{x}, \mathrm{~B}_{y}, \mathrm{~B}_{z}, \mathrm{E}_{x}, \mathrm{E}_{y}, \mathrm{E}_{z}$ are the components of magnetic and electric fields.

Depending on the geometry and the device type, external fields in the code can be defined in one of the following formats: (i) Three-dimensional tables of $\vec{E}$ and $\vec{B}$ in the input rectangular coordinate system obtained by the help of external codes. For the calculation of the field value at the particle location a quadratic interpolation routine is used. (ii) Two-dimensional tables in the plane $\{r, z\}$ for the elements with axial symmetry such as solenoids or Einzel lenses. (iii) Two dimensional tables of the $\mathrm{B}_{y}$ component of the magnetic field in the median plane $(\mathrm{x}, \mathrm{z})$ for rectangular dipole magnets and for fringe field fall-off of any dipole magnet. Off-median component $\mathrm{B}_{y}$ and components $\mathrm{B}_{x}$ and $\mathrm{B}_{z}$ are evaluated using the method described in [6]. (iv) The fringe field fall-off for dipole and multipole elements is described by a six-parameter Enge function [7]:

$$
F(z)=\frac{1}{1+\exp \left(a_{0}+a_{1}(z / D)^{1}+\ldots+a_{5}(z / D)^{5}\right)},
$$

where $\mathrm{z}$ is the distance along the line which is perpendicular to the effective field boundary, D is the full air-gap of the multipole element.

Space charge fields of multi-component ion beams are defined as the result of solving the corresponding Poisson equation. The charge distribution in the mesh-points of the rectangular mesh is defined using the "clouds-in-cell" method. The code calculates both two-dimensional (for DC beams) and three-dimensional (for bunched beams) space charge fields. The three-dimensional Poisson equation is solved with rectangular boundary conditions in the transverse direction and periodic conditions along the direction of beam propagation. The detailed description of the space charge routine can be found in ref. [8]. A special routine has been written for the integration of multi-component DC ion beams through bending magnets.

The code TRACK supports the following electromagnetic elements for acceleration, transport and focusing of multi-component ion beams:

- Any type of RF accelerating resonator with realistic 3D fields.

- Radio Frequency Quadrupoles (RFQ).

- Solenoids with fringing fields.

- Bending magnets with fringing fields.

- Electrostatic and magnetic multipoles (quadrupoles, sextupoles, ... ) with fringing fields.

- Multi-harmonic bunchers.

- Axial-symmetric electrostatic lenses.

- Entrance and exit of a high voltage (HV) deck.

- Accelerating tubes with DC distributed voltage.
- Transverse beam steering elements.

- Stripping foils or films (for RIA, not general yet).

- Horizontal and vertical jaw slits for beam collimation.

- Static ion-optics devices with both electric and magnetic realistic three-dimensional fields.

Three-dimensional interpolation of the electromagnetic fields is performed within the full aperture of ion-optics devices. For most common elements the fields can be described by several methods which makes the code TRACK compatible with many existing beam transport codes. This feature is valuable for the extraction of transfer matrices and the comparison of the simulation results with existing codes.

Prior to the simulation with the code TRACK three main steps have to be performed:

1. The elements of the accelerator system must be defined using first and, if necessary, higher order optimization codes. TRACK can also be used in some cases.

2. Preparation of field tables. This step requires the use of external codes such as Microwave studio (MWS) and Electromagnetic studio (EMS) [9]. A special preprocessor code has been written for the transformation of the calculated fields to the rectangular mesh with uniform step which is required for the code TRACK.

3. At the first passage through the accelerator the code TRACK sets phases in the SC resonators according to the user defined values. The optimization of phases using TRACK or other codes is required prior to phase setting.

\section{IMPORTANT FEATURES}

Depending on the task, the code TRACK can be compiled for the simulation of up to $10^{6}$ particles on a regular desktop PC. The baseline version of the RIA driver linac [10] contains about $400 \mathrm{RF}$ resonators operating at frequencies from $57.5 \mathrm{MHz}$ to $805 \mathrm{MHz}, 90$ solenoids, 16 bending magnets and about 100 quadrupoles. The simulation of $10^{4}$ particles on a $3 \mathrm{GHz}$ PC requires about 20 minutes. To study beam halo formation and eventual beam losses the simulation of large number of particles through multiple accelerators with randomly seeded errors is required. For these reasons, the code was parallelized using the Message Passing Interface MPI and run on the new Linux cluster JAZZ at the Laboratory Computing Resource Center of Argonne National Laboratory [11].

One of the useful features of the code TRACK is the ability to calculate the transverse and the longitudinal acceptance along the accelerator system by simulating large phase space volumes and tracking survived particles. The phase space image of the survived particles at the entrance 
of a given section of the accelerator forms the corresponding acceptance. An extensive number of beam parameters are calculated after each element and written into an output file. The list of parameters includes beam centroids; rms values and maximum beam sizes in $\mathrm{x}, \mathrm{y}$ and the phase; three types of normalized emittances in the three phase space planes. These are the rms emittances and the emittances containing $\mathrm{p} \%$ and $100 \%$ of particles, where $\mathrm{p}$ is a percentage to specify as input, for example $p=99.5$. The emittance is calculated as the area of the corresponding ellipse divided by $\pi$. The ellipse orientation is defined from the second moments of the particle distribution. The Twiss parameters and the numbers of lost and survived particles are also determined. The output data is usually treated using a scientific software for statistical analysis.

\section{BENCHMARKING}

During code development, codes like TRANSPORT, COSY, GIOS and RAYTRACE were used to check TRACK's implementation of the different beam line elements. Benchmarking was essentially done for the end-toend simulation of the RIA driver linac. The lattice consists of one or more ECR ion sources, a LEBT with a MHB and a RFQ serving as an injector to a SC linac. The linac is subdivided into three sections separated by two stripping stations with appropriate magnetic transport systems. The simulation of the low-energy part (from the ion source to the entrance of the SC linac) was compared with both DYNAMION [12] and PARMTEQ [13] simulations and found to agree reasonably well. For the SC linac, we recently performed detailed comparisons with the code IMPACT [14]. IMPACT has undergone many updates to meet the requirements of the RIA facility. Features such as multiple charge acceleration and beam stripping have been added to the code. An excellent agreement was obtained between TRACK and IMPACT for the different linac sections. These results are reported in this conference [15].

\section{RECENT UPDATES}

Since the latest studies [11], the code TRACK has undergone several updates and improvements. Among these we mention:

- The implementation of a new and fast algorithm for transverse corrective steering to compensate for misalignment errors [16].

- Separate treatment of static and dynamic errors. Static errors are associated with measurement errors and could be corrected for. Dynamic errors are associated with time fluctuations which are usually smaller than static errors and cannot be corrected for.

- The implementation of a realistic procedure to correct for static field amplitude and phase errors using a limited number of correcting cavities.
- More accurate simulation of RFQ's using an 8-term potential expansion for a regular accelerating cell and 3D field tables for a transient and radial matching cells. The field in the radial matcher can be also described by Enge function.

- Treat bending magnets with homogeneous or inhomogeneous field and arbitrary pole face.

- A matrix option of the code to be used for beam matching and optimization as well as direct comparison with other codes.

- Control particle loss for each integration step along every device and output the coordinates of lost particles in the reference frame of the corresponding device. This information is of special importance for radiation shielding calculations.

\section{REFERENCES}

[1] P.N. Ostroumov and K.W. Shepard. Phys. Rev. ST. Accel. Beams 11, 030101 (2001).

[2] K.L. Brown, The ion optical program TRANSPORT. Technical Report 91, SLAC, 1979.

[3] K.R. Crandall, TRACE 3-D Documentation, Report LA11054-MS, Los Alamos, 1987.

[4] M. Berz, COSY INFINITY, Version 8 User's Guide and Reference Manual, MSU, 1999.

[5] H. Wollnik, J. Brezina and M. Berz, Nucl. Instr. and Meth. A 258 (1987) 408.

[6] S. Kowalsky and H.A. Enge, RAYTRACE, MIT Report, Cambridge, Massachusetts, July 1, 1987.

[7] H.A. Enge, Focusing of Charged Particles, Vol. 2, p. 203, A. Septier, editor, Academic Press Inc., New York, 1967.

[8] V.A. Moiseev and P.N. Ostroumov, Proceedings of the Sixth European Part. Accel. Conf., Stockholm, Sweden, June 2226, 1998, edited by S. Myers et al, p.1216.

[9] CST Microwave Studio, User Manual Version 3.0, January 2001, CST GmbH, Darmstadt, Germany http://www.cst.de/.

[10] P.N. Ostroumov, Phys. Rev. ST. Accel. Beams, Volume 5, 030101 (2002).

[11] P.N. Ostroumov, V. N. Aseev, B. Mustapha. Phys. Rev. ST. Accel. Beams, Volume 7, 090101 (2004).

[12] A.A. Kolomiets et al, Proceedings of the Sixth European Part. Accel. Conf., Stockholm, Sweden, June 22-26, 1998, edited by S. Myers et al, p.1201.

[13] T. P. Wangler et al., " Advanced Beam-Dynamics Simulation Tools for RIA ", Proc. of LINAC 2004, Luebeck, Germany, 2004, p. 186.

[14] J. Qiang, R. D. Ryne, S. Habib, and V. Decyk, J. Comput. Phys. 163 (2000) 434.

[15] B. Mustapha et al., "RIA Beam Dynamics: Comparing TRACK to IMPACT", this conference.

[16] V.N. Aseev, E.S. Lessner and P.N. Ostroumov, "Optimization of Steering Elements in the RIA Driver Linac", this conference. 